\title{
Actividade física de crianças e jovens - haverá um declínio? Estudo transversal em indivíduos dos dois sexos dos 10 aos 19 anos de idade.
}

\author{
M. Alexandra Vasconcelos \\ José Maia \\ Faculdade de Ciências do Desporto e de Educação Física \\ Universidade do Porto \\ Portugal
}

https://doi.org/10.5628/rpcd.01.03.44

\section{RESUMO}

Os objectivos desta pesquisa de natureza transversal são os seguintes: (1) determinar a existência ou não de declínio nos níveis de actividade física das crianças e adolescentes ao longo da idade, em ambos os sexos; (2) identificar se o declínio da actividade física das raparigas é mais acentuado do que o dos rapazes; (3) verificar se os rapazes possuem níveis de actividade física superior aos das raparigas.

A amostra é constituída por 5949 alunos, 3073 do sexo feminino e 2876 do sexo masculino, com idades compreendidas entre os 10 e os 19 anos de idade, pertencentes a escolas do $2^{\circ}$ e $3^{\circ}$ ciclos do ensino básico, da Região Norte do País e da Região Autónoma dos Acores (Ilha Terceira). A actividade física foi avaliada através do Questionário de Baecke e col. (1982), que permite estimar o índice de actividade física no trabalho/escola índice de actividade física no tempo de lazer, índice de actividade física no desporto e índice de actividade física total. Os procedimentos estatísticos utilizados foram a média, desvio padrão, o t-teste e a análise de variância. O nível de significância foi mantido em $5 \%$ e o software estatístico utilizado foi o SPSS 10.0 .

Os principais resultados e conclusões foram os seguintes: (1) não se verifica a existência de declínio na actividade física das crianças e adolescentes dos dois sexos, com a excepção dos 18 para os 19 anos de idade; (2) nas raparigas verifica-se um incremento da actividade física até aos 16 anos, seguido de estabilização entre os 17-18 anos. Por sua vez, nos rapazes o incremento ocorre até aos 13 anos e o período de estabilização entre os 13-18 anos; (3) aos 18 e 19 anos o declínio da actividade física das raparigas é mais acentuado do que o dos rapazes; (4) os valores médios do índice de actividade física dos rapazes são superiores aos das raparigas em todos os escalões etários.

Palavras-chave: Actividade física, declínio, raparigas, rapazes.

\begin{abstract}
Is there a Decline in Physical Activity? A Cross-Sectional Study in Children and Youngsters of Both Gender from 10 to 19 Years old.

The purpose of this transverse study is as follows: (1) to determine the existence or not of a decline in physical activity levels in children and teenagers of both genders; (2) to identify if the girls' decline is more evident than that of boys; (3) to verify if boys have higher levels of physical activity than girls.

Sample size consists in 5949 pupils, 3073 females and 2876 males, from 10 to 19 years old, belonging to 2nd and 3rd group of basic education schools, in the North Region of Portugal, as well as the Autonomous Region of Azores (Terceira Island). Physical activity was assessed with the Baecke Questionnaire, that allows the estimative of physical activity level at work/school, physical activity level in leisure time, physical activity level at sports and the total physical activity (a sum of previous levels). Statistical procedures used were the mean, standard deviation, the $t$-test and ANOVA. Significance level was maintained in 5\% and the statistical software used was the SPSS 10.0 Major results and conclusions obtained were: (1) no decline was found in physical activity of children and teenagers of both genders, with an exception of 18 and 19 years old; (2) we verified that girls increase their physical activity till 16 years old, followed by a stable period between 17-18 years old. On the other hand, boys have an increase of their levels till 13, and stability between 13-18; (3) from 18 and 19 years the decline of physical activity in girls is more relevant than boys; (4) the mean values of physical activity level are higher for boys than for girls in all age levels.
\end{abstract}

Keywords: Physical activity, decline, girls, boys. 


\section{INTRODUÇÃO}

A inactividade física é um factor de risco de doenças cardiovasculares $(3,20,29)$ que estão na origem das principais causas de morte, de incapacidade e da ausência de qualidade de vida dos indivíduos das sociedades industrializadas (39). Não só a inactividade física como também o excesso de peso são factores comportamentais de risco para estas doenças $(4,12,39)$. Nos E.U.A., em 1994, por exemplo, as doenças cardiovasculares foram responsáveis por $45.2 \%$ de todas as mortes que aí se verificaram (12). Actualmente, existe um forte corpo de evidências salientando que a actividade física (AF) traz benefícios para a saúde $(4,7,20,29,39)$, nomeadamente no que concerne à prevenção de doenças cardiovasculares, diabetes, obesidade, hipertensão, osteoporose, desordens emocionais (depressão, ansiedade, etc... ), e alguns tipos de cancro $(3,4,26,29,39)$. Desta forma, contribui grandemente para a redução das causas de mortalidade e morbilidade das populações $(3,4,20,39)$.

Estudos têm demonstrado que em países industrializados existe um elevado número de sedentarismo na população adulta, acima de $30 \%$, e que apenas uma pequena porção realiza actividade física regular (ver, por exemplo: 20, 39). Por sua vez, Paffenbarger e Lee (29) referem valores ainda mais assustadores ao indicar que $60 \%$ da população adulta dos E.U.A. realiza pouca ou nenhuma actividade no tempo de lazer. Face ao risco significativamente elevado para um número de problemas de saúde, a inactividade física tornou-se uma das grandes preocupações da Saúde Pública $(20,39)$.

Uma das tarefas a realizar para mudar os comportamentos e hábitos de risco que afectam a saúde do indivíduo é a de incrementar a actividade física e o exercício regular na vida das pessoas. Neste sentido, urge educar a população para a saúde, encorajando-a e preparando-a para que inclua actividade física no seu quotidiano $(20,24)$. Resultados de vários estudos epidemiológicos sugerem que quantidades adequadas de exercício regular melhoram a aptidão física, preservam a saúde, aumentam a qualidade de vida e a longevidade $(3,4,7,20,29)$. Encontrar estratégias para que se aumente o gasto energético diário total de cada indivíduo pode ser o necessário para obter muitos benefícios de saúde $(12,20)$.
Mas o sedentarismo não é uma característica exclusiva dos adultos. De acordo com Sallis e Owen (39), parece que os adolescentes estão a adoptar os hábitos sedentários dos adultos, bem como a forma de encarar o exercício físico, nomeadamente as razões habituais para não o realizarem. Adoptando, os adultos, estilos de vida pouco activos e servindo de modelos para os jovens é natural que tal ocorra (2). Esta situação alerta para a necessidade de primeiro mudar os hábitos dos adultos, de modo a que mais facilmente se intervenha junto dos mais jovens $(2,24)$. Um dos factores que contribui para a sedentarização dos jovens é a redução dos esforços físicos na deslocação para a escola e nos passatempos, nomeadamente no visionamento da televisão, nos jogos electrónicos e de computador, socializar sentados, etc. Durante o seu quotidiano não realizam actividade física em quantidades e intensidades suficientes para promover efeitos benéficos sobre a saúde, nomeadamente na prevenção dos factores de risco (39). Assim, o nível de actividade física natural deste grupo é reduzida, conduzindo a uma diminuição ou deterioração da condição física, que por sua vez, se reflecte numa maior morbilidade. Esta situação leva a uma menor atracção pela actividade física e maior atracção pelas actividades de lazer passivas, surgindo, assim, um círculo vicioso de inactividade. A revisão de diversos estudos epidemiológicos indica que os níveis de AF declinam significativamente durante os anos escolares, à medida que os rapazes e raparigas passam da infância para a adolescência e até à idade adulta $(1,39)$. Quanto mais cedo se efectuar intervenções junto daqueles, maior será a probabilidade de se atrasar ou parar o declínio da AF com o avançar da idade (39).

Deste modo, para que se possa intervir de forma eficaz, interessa saber quão activas são as crianças e adolescentes e quantas é que cumprem as linhas de orientação da AF definidas pela International Consensus Conference on Physical Activity Guidelines for Adolescents (39). Para a obtenção desse conhecimento é importante que se efectuem estudos epidemiológicos descritivos da AF dos jovens, uma vez que existem muito poucos dados sobre esta população quando comparada com a dos adultos $(10,39)$. Esta será a base para expandir as políticas e os esforços para educar os jovens sobre os benefícios da AF e os pré- 
-requisitos para se ser mais activo, ajudando-os, deste modo, a criar as fundações para uma vida fisicamente activa $(2,10)$.

Nos estudos que consultamos, efectuados na população portuguesa $(9,15,17,32,35,40,46)$ em que esta temática foi de algum modo abordada, não é clara a resposta inequívoca à presença ou ausência de declínio da actividade física em função da idade. Daqui a justificação desta pesquisa, sobretudo no ordenamento da diversidade de resultados relativos a esta questão.

Com o intuito de contribuir para um maior conhecimento dos níveis de AF das crianças e jovens do nosso país, mais especificamente da Região Norte e da Região Autónoma dos Açores, e assim dar continuidade a estudos já efectuados em Portugal, surge esta pesquisa transversal. No entanto, será nossa principal preocupação determinar a existência ou ausência de declínio nos níveis de actividade física das crianças e adolescentes ao longo da idade, em ambos os sexos, bem como verificar se os rapazes são mais activos do que as raparigas.

\section{METODOLOGIA}

\section{Amostra}

A amostra é constituída por rapazes $(n=2876)$ e raparigas $(n=3073)$ com idades compreendidas entre os 10 e os 19 anos, pertencentes aos $2^{\circ}$ e $3^{\circ}$ ciclos do Ensino Básico e Ensino Secundário. Os dados foram recolhidos através de questionários distribuídos por diversas escolas $\mathrm{C}+\mathrm{S}$ e Secundárias da Região Norte do País, nomeadamente no Porto, Viseu, Vila Real e Bragança, e na Ilha Terceira (Região Autónoma dos Açores), abrangendo um total de 5949 alunos, do $5^{\circ}$ ao $12^{\circ}$ anos de escolaridade. É importante salientar que só foi possível uma amostra desta dimensão pela recolha que tem vindo a ser efectuada ao longo dos últimos 3 anos por diversos alunos de mestrado desta Faculdade $(9,15$, $17,32,35,46)$.

\section{Instrumento}

Para avaliar a AF habitual foi utilizado o Questionário de Baecke e col. (1982).

Trata-se de um questionário composto por três partes distintas: o primeiro grupo de questões procura determinar o índice de AF na escola (IAFE); o segundo grupo é dedicado às actividades desportivas (IAFD); a última parte visa avaliar a AF nos tempos de lazer (IAFL). Cada uma destas partes fornece os dados necessários para estimar os três índices de AF, cujo somatório permite determinar o valor da $\mathrm{AF}$ total (IAFT).

O questionário é constituído por dezasseis itens, a partir dos quais se calculam os índices atrás mencionados. As questões são fechadas e de múltipla escolha, obedecendo à escala de valores de Lickert, em que as respostas estão codificadas de 1 a 5 , segundo ordem crescente de importância.

A classificação das actividades foi efectuada de acordo com a tabela de códigos para o Questionário de Baecke e col. (1982) modificado, ou seja, uma classificação em função do tipo de actividade, da frequência semanal, do número de meses em que se realiza e da intensidade. Salientamos que neste estudo é considerado exclusivamente a actividade física total, isto é, o somatório dos três índices mencionados anteriormente, com um intervalo de variação entre 1 e 17. Em Portugal foram realizados diversos estudos (ver por exemplo: 9, 17 e 35) em que se determinou a fiabilidade deste questionário, cujos valores de correlação variaram entre 0.60 e 0.98 . Estes resultados confirmam a consistência elevada das respostas ao questionário, mesmo quando utilizado em contextos com alguma diferença sociocultural.

\section{Procedimentos estatísticos}

A descrição dos dados segue os procedimentos habituais - médias e desvios padrão. A estatística inferencial contém o t-teste e a análise de variância. O nível de significância foi mantido em $5 \%$ e o software estatístico utilizado foi o SPSS 10.0.

\section{RESULTADOS}

\section{Actividade Física das raparigas}

No quadro 1 e na figura 1 estão as estatísticas descritivas fundamentais do índice de actividade física (IAF) das raparigas entre os 10 e os 19 anos. 
Quadro 1: Valores da média (M), desvio padrão (dp), mínimo (Min.) e máximo (Máx.] do IAF das raparigas em função da idade.

\begin{tabular}{lcccc} 
Idade & Amostra & $\mathrm{M} \pm \mathrm{dp}$ & Min. & Máx. \\
\hline 10 & 141 & $7.41 \pm 1.17$ & 3.00 & 9.75 \\
11 & 362 & $7.31 \pm 1.33$ & 2.25 & 10.63 \\
12 & 369 & $7.53 \pm 1.18$ & 3.00 & 11.13 \\
13 & 399 & $7.52 \pm 1.20$ & 3.25 & 11.25 \\
14 & 443 & $7.58 \pm 1.22$ & 3.00 & 11.00 \\
15 & 370 & $7.81 \pm 1.21$ & 3.75 & 11.63 \\
16 & 402 & $8.09 \pm 1.19$ & 4.75 & 10.88 \\
17 & 353 & $7.92 \pm 1.29$ & 3.13 & 10.88 \\
18 & 162 & $7.92 \pm 1.26$ & 4.38 & 10.63 \\
19 & 52 & $7.65 \pm 1.22$ & 5.50 & 10.25
\end{tabular}

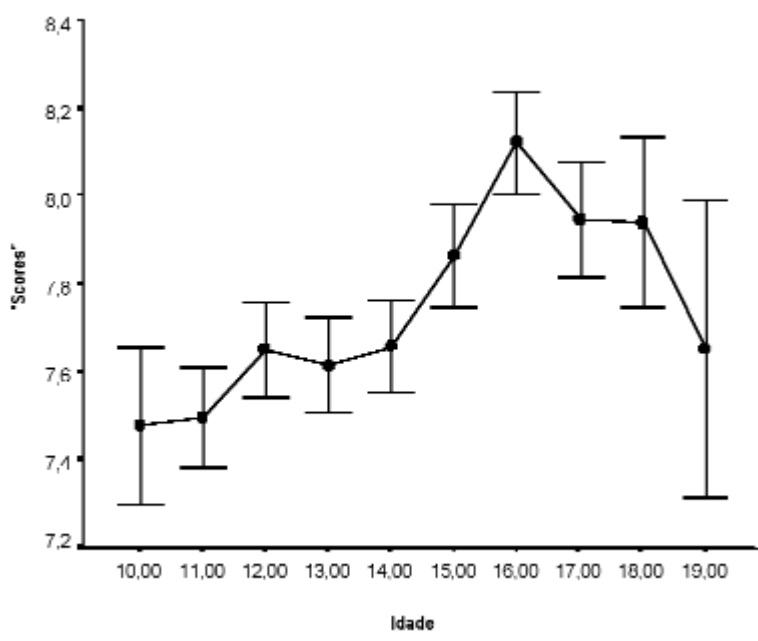

Figura 1: Comportamento dos valores médios do índice de actividade física das raparigas em função da idade.

Os dados sugerem a existência de diferentes comportamentos nos valores das médias do IAF ao longo da idade. Verifica-se uma oscilação na curva do IAF, sendo marcante um incremento até aos 16 anos (de 7.41 até 8.09); a partir daqui ocorre um decréscimo no referido índice (8.09 até 7.65), com uma estabilização dos 17 aos 18 anos.

A análise da variância evidenciou diferenças significativas nas médias ao longo da idade $(\mathrm{F}=13.65, \mathrm{p}$ $=.000$ ), salientando o escalão 15-18 anos relativamente aos 10, 11, 12, 13 e 14 anos.
As raparigas com 19 anos são as únicas cujo IAF não apresenta diferenças significativas comparativamente a qualquer outra idade desta amostra.

\section{Actividade Física dos rapazes}

O quadro 2 e a figura 2 referem-se às estatísticas descritivas fundamentais do IAF dos 10 aos 19 anos de idade, nos rapazes.

Quadro 2: Valores da média (M), desvio padrão (dp), mínimo (Min.) e máximo (Máx.) do IAF dos rapazes em função da idade.

\begin{tabular}{lcccc} 
Idade & Amostra & $M \pm d p$ & Min. & Máx. \\
\hline 10 & 104 & $7.68 \pm 1.06$ & 4.75 & 10.25 \\
11 & 263 & $8.23 \pm 1.13$ & 4.88 & 11.75 \\
12 & 334 & $8.46 \pm 1.21$ & 4.63 & 11.63 \\
13 & 298 & $8.49 \pm 1.19$ & 4.25 & 11.38 \\
14 & 321 & $8.42 \pm 1.13$ & 3.88 & 11.25 \\
15 & 442 & $8.43 \pm 1.12$ & 4.25 & 12.00 \\
16 & 481 & $8.54 \pm 1.13$ & 4.00 & 12.00 \\
17 & 389 & $8.53 \pm 1.09$ & 5.88 & 11.25 \\
18 & 161 & $8.65 \pm 1.16$ & 6.13 & 11.63 \\
19 & 55 & $8.52 \pm 1.16$ & 5.38 & 10.75
\end{tabular}

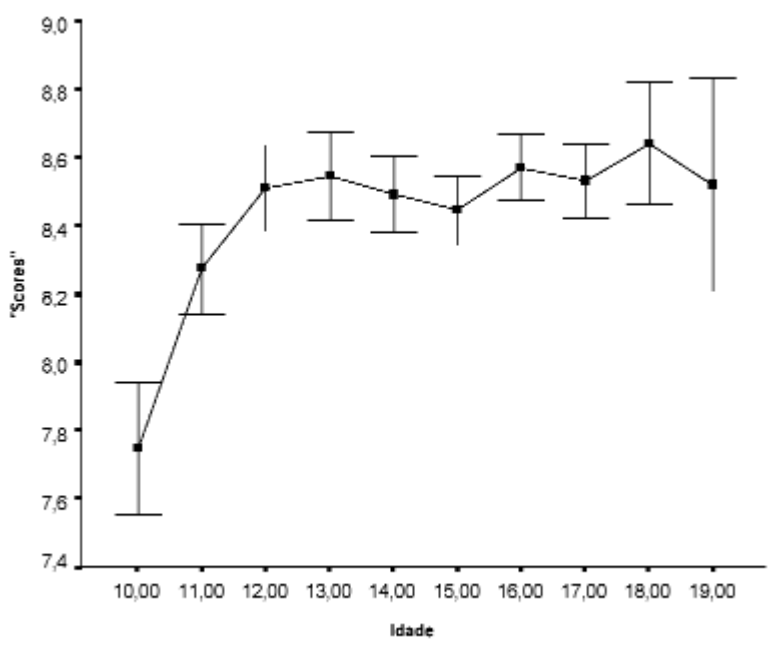

Figura 2: Comportamento dos valores médios do índice de actividade física dos rapazes em função da idade. 
Da análise da figura e do quadro supracitados, podemos constatar que o IAF dos rapazes no escalão etário considerado é marcado por dois grandes momentos: um primeiro momento de incremento e posteriormente uma estabilização.

Do estudo do comportamento dos rapazes ao longo da idade constatamos que a média inicial do IAF foi de 7.68, tendo sofrido um incremento até aos 13 anos $(M=8.49)$, estabilizando de seguida, apesar de um incremento substancial aos 18 anos (de 8.53 para 8.65).

Através da análise de variância constatou-se a existência de diferenças significativas nas diversas classes de idade $(\mathrm{F}=7.61, \mathrm{p}=.000)$. Essas diferenças ocorrem exclusivamente entre os 10 anos e todas as outras idades consideradas.

\section{Comparação dos níveis de Actividade Física entre raparigas e rapazes}

No quadro 3 é bem visível a existência de diferenças significativas $(\mathrm{p}<0.05)$ no IAF entre raparigas e rapazes para todas as idades, com vantagem nítida para os rapazes, com a excepção dos 10 anos em que $\mathrm{p}=0.067$.

Quadro 3: Valores das médias ( $M$ ], desvios padrão (dp), $t$ [t-teste) e $p$ [nível de significância) dos IAF das raparigas e dos rapazes.

\begin{tabular}{lcccc} 
Idade & $\begin{array}{c}\text { Raparigas } \\
\mathrm{M} \pm \mathrm{dp}\end{array}$ & $\begin{array}{c}\text { Rapazes } \\
\mathrm{M} \pm \mathrm{dp}\end{array}$ & $\mathrm{t}$ & $\mathrm{p}$ \\
\hline 10 & $7.41 \pm 1.17$ & $7.68 \pm 1.06$ & -1.84 & .067 \\
11 & $7.31 \pm 1.33$ & $8.23 \pm 1.13$ & -9.03 & .000 \\
12 & $7.53 \pm 1.18$ & $8.46 \pm 1.21$ & -10.29 & .000 \\
13 & $7.52 \pm 1.20$ & $8.49 \pm 1.19$ & -10.66 & .000 \\
14 & $7.58 \pm 1.22$ & $8.42 \pm 1.13$ & -9.65 & .000 \\
15 & $7.81 \pm 1.21$ & $8.43 \pm 1.12$ & -7.59 & .000 \\
16 & $8.09 \pm 1.19$ & $8.54 \pm 1.13$ & -5.76 & .000 \\
17 & $7.92 \pm 1.29$ & $8.53 \pm 1.09$ & -6.91 & .000 \\
18 & $7.92 \pm 1.26$ & $8.65 \pm 1.16$ & -5.37 & .000 \\
19 & $7.65 \pm 1.22$ & $8.52 \pm 1.16$ & -3.78 & .000
\end{tabular}

O valor médio mais elevado das raparigas é aos 16 anos $(M=8.09)$ e o mais baixo aos 11 anos $(M=7.31)$. Por sua vez, os rapazes apresentam a sua média mais elevada aos 18 anos $(\mathrm{M}=8.65)$ e a mais baixa aos 10 anos $(M=7.68)$.
Enquanto que para os rapazes o incremento do IAF é mais notório dos 10 aos 13 anos, nas raparigas esta fase ocorre entre os 13 e os 16 anos. A partir desta idade as raparigas apresentam decréscimo nos seus índices, o mesmo não se verificando para os rapazes.

\section{DISCUSSÃO}

Após análise dos resultados obtidos, relativamente ao IAF, constatamos que na nossa pesquisa, contrariamente ao que é comummente referido na literatura, não parece existir um declínio na AF das crianças e adolescentes de ambos os sexos antes dos 19 anos. As raparigas apresentam um incremento no IAF até aos 16 anos, entre os 17 e 18 há uma estabilização e o declínio inicia-se aos 19 anos. No caso dos rapazes observou-se um incremento substancial até aos 13 anos, seguido de uma estabilização até aos 18 anos e aos 19 anos um declínio do IAF.

$O$ incremento da AF pode ser parcialmente explicado por uma maior adesão a actividades desportivas nos tempos livres e na escola e a uma menor adesão ao sedentarismo. A entrada nos $2^{\circ}$ e $3^{\circ}$ ciclos pelos jovens implica mais aulas de Educação Física, mais actividades realizadas na escola em que a componente desportiva está presente, a possibilidade de uma maior adesão a clubes e grupos desportivos conjuntamente com os colegas e amigos, factores que podem determinar o IAF. No caso específico das raparigas, acrescem ainda as preocupações de ordem estética que se evidenciam nestas fases.

As influências supracitadas sobre a AF vão de encontro à perspectiva defendida pelos Modelos Ecológicos - em que os factores interpessoais e institucionais constituem determinantes da prática da $\mathrm{AF}$-, uma das teorias desenvolvidas no sentido de facilitar o estudo das variáveis que se crê estarem relacionadas com a AF (39).

O período de estabilização nos rapazes pode resultar da dificuldade existente em incrementar substancialmente as médias já existentes, uma vez que são as mais elevadas do IAF. Na situação das raparigas, a estabilização reporta-se apenas ao escalão etário 17 -18 anos e pode ser consequência de um aumento de responsabilidades familiares, das responsabilidades ao nível dos estudos ou de uma maior adesão a comportamentos sedentários comparativamente às rapa- 
rigas mais novas, como defendem Bradley, McMurray, Harrel e Deng (8).

O declínio evidenciado aos 19 anos, em ambos os sexos, pode ser consequência de um aumento dos comportamentos sedentários $(1,27)$, nomeadamente, de um aumento do tempo despendido a assistir televisão, a jogar no computador (36), a "viajar" na internet, e de uma maior responsabilidade resultante da entrada na Faculdade, da aquisição de novos papeis e funções familiares, da entrada no mundo do trabalho para alguns dos jovens, ou simplesmente um artificio da reduzida dimensão da amostra $(n=$ 52 nas raparigas e $\mathrm{n}=55$ nos rapazes). Garcia, Broda, Frenn, Coviak, Pender e Ronis (16) consideram que os factores anteriormente referidos, sobretudo o penúltimo, podem resultar de um usufruto menor ao nível de apoio social e de modelos para o exercício, dos adolescentes mais velhos relativamente aos mais jovens. Na sua perspectiva, uma das estratégias eficazes para encorajar o aumento do exercício nos dois géneros sexuais durante a infância e adolescência consiste numa maior exposição a modelos fisicamente activos, que estabeleçam regras para se exercitarem e que forneçam um apoio emocional e material para se ser activo. Esta posição está em acordo com a Social Cognitive Theory, em que o incentivo e reforço para a prática da AF são considerados factores determinantes da mesma.

Face ao exposto podemos afirmar que os dados da nossa pesquisa não estão de acordo com os estudos consultados, em que se observou uma diminuição na AF com o aumento da idade $(11,21,25,27,34,38$, 42, 43, 45).

Caspersen, Pereira e Curran (11) apuraram que é entre os 15-18 anos que os adolescentes, de ambos os sexos, correm maior risco de diminuição da AF. Para este escalão etário, a diminuição anual de 5 dos 10 padrões de actividade específicas consideradas foi superior a 0.5 pontos percentuais. Houve um incremento ao nível da inactividade de 4.2 pontos percentuais para os rapazes e de 3.0 pontos percentuais para as raparigas. No nosso caso, os adolescentes só apresentaram uma diminuição da AF aos 19 anos. Entre os 15 e os 18 anos verificaram-se apenas fases de estabilização para os rapazes e de incremento da AF para as raparigas.
Van Mechelen e Kemper (43) consideram que os níveis de AF decrescem gradualmente dos 13 aos 16 anos em ambos os sexos e de forma menos gradual dos 16 aos 21 anos.

Em 1993, Sallis (37) constatou que durante a vida escolar a AF declinava cerca de $2.7 \%$ por ano nos rapazes e $7.4 \%$ nas raparigas. Esta posição foi igualmente defendida por Armstrong (1), que após revisão da literatura concluiu que o declínio dos níveis de actividade das raparigas era superior ao dos rapazes. Numa direcção oposta encontramos uma revisão de estudos em animais e humanos sobre o declínio da AF efectuado, novamente, por Sallis (38). Desta vez, os resultados apresentados levaram o autor a entender que o declínio da AF se trata de um fenómeno mais acentuado entre os 13 -18 anos e superior nos rapazes em comparação com as raparigas.

Para Telama e Yang (42) é a partir dos 12 anos que ocorre um decréscimo marcante na frequência da AF. Na nossa amostra é precisamente nesta fase que ocorrem os incrementos mais significativos, para ambos os sexos. Na sua pesquisa, a maioria do declínio na AF ocorreu entre os 12-18 anos para os rapazes e entre os 12-15 para as raparigas. Consideram ainda que o declínio é mais evidente nos rapazes (2.2\% por ano) do que nas raparigas ( $1.4 \%$ por ano) e que estas aderiram mais frequentemente à AF após os 18 anos.

Também Kimm, Glynn, Kriska, Fitzgerald, Aaron, Similo, McMahon e Barton (21) observaram uma diminuição da AFT durante a transição da infância para a adolescência, numa amostra constituída unicamente por raparigas. Os níveis de AF diária declinaram 35\% dos 9-10 anos até aos 18-19 anos, enquanto os níveis de AF habitual declinaram $83 \%$. Das pesquisas consultadas encontramos duas em que se observou declínio da AF apenas nos sujeitos do sexo masculino.

Para Van Mechelen, Twisk, Post e Kemper (44) a AF semanal diminui apenas nos rapazes, entre os 13-27 anos. Durante um período de 15 anos apresentaram um decréscimo da AF de $31 \%$, de 10.6. s -1 passaram para 7.3. s -1 . Por sua vez, as raparigas tiveram uma redução de $8 \%$ na $\mathrm{AF}$, de 9.1. s -1 para 8.4. s -1 , valor que não foi significativo ( $p>0.05)$. Uma análise mais detalhada mostrou que nas raparigas 
ocorreu um decréscimo de 3\% na AF dos 13 aos 16 anos, seguido de um incremento de $5 \%$ entre os 16 -21 e entre os 21-27 um novo decréscimo - $10 \%$. Nos rapazes durante esses mesmos períodos verificou-se uma diminuição de $20 \%$, um incremento de $5 \%$ e um decréscimo de $16 \%$.

Boreham, Twisk, Savage, Cran e Strain (5) constataram a existência de um pequeno decréscimo (-20\%) na $\mathrm{AF}$ apenas nos rapazes. No caso das raparigas, o declínio ocorreu ao nível da participação no desporto. Como podemos constatar, na nossa amostra o período de estabilização é maior nos rapazes do que nas raparigas e situa-se entre os 13-18 anos.

Os estudos que encontramos em que foi focada a estabilização da AF não são unânimes quanto à fase da vida em que esta é mais marcante. Para Pate, Baranowski, Dowda e Trost (30), a AF estabiliza durante a infância. O nível de AF da sua pesquisa foi constante durante o período de observação, o que se confirma pelo facto de a média do index PAHR-50 se ter alterado pouco de ano para ano - subiu de 12 para $15 \%$ em três anos. Não encontraram diferenças significativas entre sexos. Contudo, os autores argumentam que a AF foi avaliada apenas em crianças, durante três anos e com duas a quatro observações por ano entre as 15.00 - 18.00 horas, o que pode condicionar os resultados obtidos.

Janz, Dawson e Mahoney (19) consideram que a AF estabiliza desde a infância até à adolescência, enquanto Malina (23) defende que este fenómeno ocorre durante a adolescência, da adolescência até ao estado adulto e ao longo das várias idades desta fase. As diferenças entre o nosso estudo e os anteriormente referidos não devem ser analisadas primordialmente à luz da questão metodológica uma vez que, quase todos, recorreram a questionários sobre a AF habitual para a aquisição de dados.

Verificamos, ainda, que os rapazes são efectivamente mais activos do que as raparigas em todas as idades. Estes resultados estão em consonância com os da literatura consultada $(1,5,8,11,16,27,34,37,38$, $41,44,45)$.

A diferença no IAF entre rapazes e raparigas pode resultar da menor auto-estima e menor passado desportivo, bem como de uma percepção do estado de saúde e auto-esquema de exercício inferiores por parte das raparigas. Para além disso, a concepção do corpo e das capacidades individuais e a atitude face à AF, podem influenciar a predisposição psicológica para a actividade (23). As transformações rápidas ao nível do auto-conceito, sentido de valia e da imagem corporal que ocorrem na adolescência, também não devem ser ignoradas, pois são fenómenos que podem afectar a AF de modo diferenciado ao longo dos vários estádios de desenvolvimento (16). Para Telama e Yang (42) os rapazes são mais activos do que as raparigas apenas aos 9 e 12 anos, enquanto que para Verschuur e Kemper (45) isso acontece apenas até aos 14/15 anos. Os autores entendem que esta diferença diminui linearmente com o aumento da idade, embora para Telama e Yang (42) após os 15 anos a diferença passe a não ser significativa.

Também Sunnegardh, Bratteby, Sjolin, Hagman e Hoffstedt (41) obtiveram resultados indiciadores de um IAF superior nos rapazes comparativamente às raparigas, e das crianças mais jovens comparativamente às mais velhas. Este último dado é contrário ao nosso uma vez que as crianças mais novas, 10 anos, apresentam valores da média de AF inferiores aos dos mais velhos, 19 anos, apesar destes já apresentarem declínio nesse índice.

Os resultados do nosso estudo indicam que o índice de actividade física revela variações nos seus valores médios de acordo com o género sexual em questão, apresentando um declínio para ambos os sexos somente a partir dos 18 anos.

\section{CONCLUSÃO}

Em conclusão, não verificamos a existência de declínio nos níveis de actividade física das raparigas e rapazes com idades compreendidas entre os 10-17 anos, mas constatamos este fenómeno dos 18 para os 19 anos. Esta fase de declínio é mais acentuada nas raparigas do que nos rapazes.

As raparigas apresentam um incremento da actividade física até aos 16 anos e os rapazes até aos 13.

Detectamos uma fase de estabilização entre os 17-18 anos nas raparigas e entre os 13-18 anos nos rapazes. Constatamos, ainda que os rapazes apresentam valores médios da actividade física superiores aos das raparigas em todas as idades, à excepção dos 10 anos de idade. 


\author{
CORRESPONDÊNCIA \\ José Maia \\ Laboratório de Cineantropometria \\ Faculdade de Ciências do Desporto \\ e de Educação Física \\ Universidade do Porto \\ Rua Dr. Plácido Costa, 91 \\ 4200 - 450 Porto \\ Portugal \\ jmaia@fcdef.up.pt
}

\section{BIBLIOGRAFIA}

1. Armstrong N (1998). O papel da escola na promoção de estilos de vida activos. In: Omniserviços. A educação para a saúde. O papel da educação física na promoção de estilos de vida saudáveis. Lisboa: 5-17.

2. Armstrong N, Welsman J (1997). Young people and physical activity. New York: Oxford University Press.

3. Blair S (1993). 1993 C. H. McCloy research lecture: physical activity, physical fitness, and health. Research Quarterly for Exercise and Sport 64, $\mathrm{n}^{\circ}$. 2: 146-158.

4. Blair S, Brodney S (1999). Effects of physical inactivity and obesity on morbidity and mortality: current evidence and research issues. Medicine and Science in Sports and Exercise 31, $\mathrm{n}^{\circ} .11$ : S646-S662.

5. Boreham C, Twisk J, Savage M, Cran G, Strain J (1997). Physical activity, sports participation, and risk factors in adolescents. Medicine and Science in Sports and Exercise 29, $\mathrm{n}^{\circ}$. 6: 788793.

6. Bouchard C, Malina R, Pérusse L (1997). Genetics of fitness and physical performance. Champaign. Illinois: Human Kinetics. 7. Bouchard C, Shephard R, Stephens T (1994). Physical activity, fitness and health: International Proceedings and Consensus Statement. Champaign. Illinois: Human Kinetics Publishers.

8. Bradley C, McMurray R, Harrel J, Deng S (2000). Changes in common activities of 3rd through 10th graders: the CHIC Study. Medicine and Science in Sports and Exercise 32, $\mathrm{n}^{\circ}$. 12: 20712078.

9. Cardoso M (2000). Aptidão física e actividade física da população escolar do Distrito de Vila Real. Estudo em crianças e jovens de ambos os sexos dos 10 aos 18 anos de idade. Dissertação de Mestrado. FCDEF-UP. Porto.

10. Caspersen C, Nixon P, Durant R (1998). Physical activity Epidemiology applied to children and adolescents. Exercise and Sport Sciences Review 26: 341-403.

11. Caspersen C, Pereira M, Curran K (2000). Changes in physical activity patterns in the United States, by sex and crosssectional age. Medicine and Science in Sports and Exercise 32, $n^{\circ} .9$ : 1601-1609.

12. Colditz G (1999). Economic costs of obesity and inactivity. Medicine and Science in Sports and Exercise 31, $\mathrm{n}^{\circ}$ 11: S663-S667. 13. Constantino J (1998). A actividade física e a promoção da saúde das populações. In: Omniserviços. A educação para a saúde. O papel da educação física na promoção de estilos de vida saudáveis. Lisboa: 20-38.

14. Dishman R, Sallis J (1994). Determinants and interventions for physical activity and exercise. In: Bouchard, C.; Shephard, R.; Stephens, T. (eds). Physical activity, fitness and health: Consensus Statement. Champaign. Illinois: Human Kinetics Publishers, 214-238.

15. Ferreira J (1999). Aptidão física, actividade física e saúde da população escolar do Centro da Área Educativa de Viseu. Estudo em crianças e jovens de ambos os sexos dos 10 aos 18 anos de idade. Dissertação de Mestrado. FCDEF-UP. Porto. 16. Garcia A, Broda M, Frenn M, Coviak C, Pender N, Ronis D (1995). Gender and developmental differences in exercise beliefs among youth and prediction of their exercise behavior. Journal of School Health 65, $\mathrm{n}^{\circ}$. 6: 213-219.

17. Henriques S (2000). Relação multivariada entre actividade física habitual e aptidão física. Uma pesquisa em crianças e jovens do sexo feminino do $6^{\circ}$ ao $9^{\circ}$ ano de escolaridade. Dissertação de Mestrado. FCDEF-UP. Porto. 
18. Ingram D (2000). Age-related decline in physical activity: generalization to nonhumans. Medicine and Science in Sports and Exercise 32, $\mathrm{n}^{\circ}$ 9: 1623-1629.

19. Janz K, Dawson J, Mahoney L (2000). Tracking physical fitness and physical activity from childhood to adolescence: the Muscatine study. Medicine and Science in Sports and Exercise 32, $\mathrm{n}^{\circ}$ 7: $1250-1257$

20. Killoron A, Fentem P, Caspersen C (1994). Moving On. International perspectives on promoting physical activity. London: Killoron, A., Fentem, P. e Caspersen, C. (eds). Health Education Authority.

21. Kimm S, Glynn N, Kriska A, Fitzgerald S, Aaron D, Similo S, McMahon R, Barton B (2000). Longitudinal changes in physical activity in a biracial cohort during adolescence. Medicine and Science in Sports and Exercise 32, $\mathrm{n}^{\circ}$ 8: 1445-1454.

22. Macera C, Pratt M (2000). Public health surveillance of physical activity. Research Quarterly for Exercise and Sport 71, $\mathrm{n}^{\circ}$. 2: 97-103.

23. Malina R (1996). Tracking of physical activity and physical fitness across the lifespan. Research Quarterly for Exercise and Sport 67 (supl.), $\mathrm{n}^{\circ}$. 3: 48-57.

24. Marques A (1998). Actividade física e saúde. A perspectiva pedagógica. In: Omniserviços. A educação para a saúde. O papel da educação física na promoção de estilos de vida saudáveis. Lisboa, 81107.

25. McKenzie T, Sallis J, Elder J, Berry C, Hoy P, Nader P, Zive $\mathrm{M}$, Broyles S (1997). Physical activity levels and prompts in young children at recess: a two-year study of a bi-ethnic sample. Research Quarterly for Exercise and Sport 68, $n^{\circ}$. 3: 195-202. 26. Montoye H, Kemper H, Saris W, Washburn R (1996). Measuring physical activity and energy expenditure. Champaign. Illinois: Human Kinetics Publishers.

27. Myers L, Strikmiller P, Webber L, Berenson G (1996). Physical and sedentary activity in school children grades 5-8: the Bogalusa Heart Study. Medicine and Science in Sports and Exercise 28, no 7: 852-859.

28. Paffenbarger R, Kampert J, Lee I, Hyde R, Leung R, Wing A (1994). Changes in physical activity and other lifeway patterns influencing longevity. Medicine and Science in Sports and Exercise 26, no 7: 857-865.

29. Paffenbarger R, Lee I (1996). Physical activity and fitness for health and longevity. Research Quarterly for Exercise and Sport 67 (Supl.), $\mathrm{n}^{\circ}$ 3: $11-28$.

30. Pate R, Baranowski T, Dowda M, Trost S (1996). Tracking of physical activity in young children. Medicine and Science in Sports and Exercise 28, n ${ }^{\circ}$ 1: 92-96.

31. Pate R, Long B, Heath G (1994). Descriptive Epidemiology of physical activity in adolescents. Pediatric Exercise Science 6: 434-447.

32. Pereira $P$ (1999). Influência parental e outros determinantes nos níveis de actividade física. Um estudo em jovens do sexo feminino dos 12 aos 19 anos. Dissertação de Mestrado. FCDEF-UP. Porto.

33. Philippaerts R (1998). Physical activity and physical fitness in male adolescents and adults. Dissertação de Doutoramento. Faculdade de Educação Física e Fisioterapia. Universidade Católica de Lovaina. Lovaina.

34. Raitakari O, Taimela S, Porkka K, Leino M, Telama R, Dahl M, Viikari J (1996). Patterns of intense physical activity among 15 to 30 year old Finns. The cardiovascular risk in Young Finns Study. Scandinavian Journal of Medicine and Science in Sports, 6: 36 39.
35. Rodrigues M. (2001). Aptidão física e actividade física habitual. Estudo em crianças e jovens de ambos os sexos do $6^{\circ}$ ao $12^{\circ}$ ano de escolaridade da Ilha Terceira, Região Autónoma dos Açores. Dissertação de Mestrado. FCDEF-UP. Porto.

36. Rowland T (1990). Exercise and children's health. Champaign. Illinois: Human Kinetics Books.

37. Sallis J (1993). Epidemiology of physical activity and fitness in children and adolescents. Critical Reviews in Food Science and Nutrition 33: 403-408.

38. Sallis J (2000). Age-related decline in physical activity: a synthesis of human and animal studies. Medicine and Science in Sports and Exercise 32, n ${ }^{\circ}$ 9: 1598-1600.

39. Sallis J, Owen N (1999). Physical activity and behavioral medicine. London: Sage publications.

40 Santos M (2000). Avaliação da actividade física habitual em crianças e jovens do Grande Porto. Dissertação de Mestrado. FCDEF-UP. Porto.

41. Sunnegardh J, Bratteby L, Sjolin S, Hagman U, Hoffstedt A (1985). The relation between physical activity and energy intake of 8 and 13 year old children in Sweden. In: Binkhorst, R.; Kemper, H.; Saris, W. (eds). Children and exercise XI. Champaign. Illinois: Human Kinetics, 183-193.

42. Telama R, Yang X (2000). Decline of physical activity from youth to young adulthood in Finland. Medicine and Science in Sports and Exercise 32, $\mathrm{n}^{\circ} .9$ : 1617-1622.

43. Van Mechelen W, Kemper H (1995). Habitual physical activity in longitudinal perspective. In: Kemper, $\mathrm{H}$. (ed.). The Amsterdam growth study: a longitudinal analysis of health, fitness, and lifestyle. Champaign. Ilinois: Human Kinetics,135-158. 44. Van Mechelen W, Twisk J, Post G, Kemper H (2000). Physical activity of young people: the Amsterdam longitudinal growth and health study. Medicine and Science in Sports and Exercise 32, $\mathrm{n}^{\circ}$. 9: 1610-1616.

45. Verschuur R, Kemper H (1985). Habitual physical activity in Dutch teenagers measured by heart rate. In: Binkhorst, R.; Kemper, H.; Saris, W. (ed.). Children and exercise XI. Champaign. Ilinois: Human Kinetics, 194-202.

46. Victor H (1999). Percepção do estado geral de saúde e actividade física. Dissertação de Mestrado. FCDEF-UP. Porto. 